\title{
Documentos
}

\section{DIALOGO ENTRE DOS CONTINENTES (CLUB DE ROMA Y FORO LATINOAMERICANO - ROMA 1976)}

Comte Promoror: Senador Profesor Amintore Fanfani, Presidente honorario del Instituto Italo-Latino Amcricano; Linbajador Norberto Trexino Zapata, Presidentc del Instituto Italo-Latino Amcricano - Delegado de México; Doclor Ga. briel Valdes, Presidente del Foro Latino-Americano; Doctor Aurelio Peccei, Presidentc del Club de Roma; Embajador Tincenzo Tornetta, Secretario Gencral del Instituto Italo-Latino Americano.

\section{DOCUMENTO FINAL}

1. Del 1 al 3 de febrero de 1976 se celebró en Roma, en la sede del Instituto ItaloLatino Americano el primer encuentro entre representantes del "Club de Roma" y del "Foro Latino-Americano", con el objeto de cxaminar las propucstas presentadas por las dos entidades, rclacionadas con la adopción de un nuevo orden econónico internacional.

2. El Instituto Italo-Latino Americano -organización intergubernamental, en ia que participan con Italia veinte Repúblicas latino-americanas- en el marco de sus finalidacics institucionales promovió $y$ organizó el encuentro entre el "Club de Roma" y' el "Foro Latino Americano". Descle su fundación el IILA se ha propuesto como objetivo principal la promoción de contactos entre representantes de todos sus Listaclos miembros, y a través de Italia, entre América Latina $y$ Europa en particular, la Comunidad Económica Europea.

3. Participaron en el "Diálogo entre dos Continentes" miembros del "Club de Roma" y del "Foro Latino-Americano"; asisticron observadores de organismos y entidades internacionalcs yr nacionales.

4. Las labores de la reunión se iniciaron con las exposiciones del Presidente honorario del IILA, Scnador Amintore Fanfani; del Presidente del Instituto, Embajador Norbcrto Treviño Zapata; del Presidente del Foro Latino-Americano, doctor Gabriel Valdés y' del P'residente del Club de Roma, doctor Aurelio Peccei. La dirección de los debates esturo a cargo del Secretario General del IILA, Embajador Vincenzo Tornetra. 
5. Los participantes señalaron que el encuentro tuvo especial importancia en vista de que, en estos momentos y en diferentes foros internacionales, se cstán llevando a calso discusiones y negocinciones con el fin de definir un "nuevo orden intemacional", sobre todo en el campo de hs relaciones económicas entre los Ëstados, especialnuente cnte los paises inchustrializados y aquellos en vías de desarrollo.

6. En cste contexto adquieren importancia particular las Resoluciones de las Naciones Unidas en la VI $y$ VII sesión especial de la Asamblea, asi como la Con[erencỉa sobre la Cooperación Económica Internacional que se estai efectuando cn Paris y la IV UNCTAD por celebrarse en Nairobi en najo de 1976. En efecto, los mecanismos que durante las tiltimis décadas han regulado las relaciones entre los Estaclos en diversos grados de clesarrollo, a menudo, han funcionado en faror de los paises inclustrializaclos y necesitan ser revisados pues existc consenso de quc, dada la complejiclad de los problemas del mundo actual, ninguno pucda enfrentarlos y resolverlos de mancral aislada.

7. En vista de que la interdependencia entre los diferentes paises es cacla dia más profunda, los sistemas de colaboración e integración deberian ser mejor organizados y mís equitativos. Es por esta razón que encucntros informales, cumo el presente "Dílogo entre dos Continentes", representan una nueva fuente de idcas y de orientaciones para la acción y adquicren un significado particular.

8. Como ha observado el Presiclente honorario del InLAl en su intervención: "cl mundo se encuentra ante tres grupos de problemas. El primero se refiere a la contaminación del ambiente $y$ n la disminución de los recursos naturales, delsilo al excesivo crecimiento demográfico, al progreso material y tecnolígico. El segundo problema toca la intensidad cuantitativa $y$ cualitiliva de Jas relaciones económicas $y$ la multiplicidad $y$ distintas potencialidades de los centros directivos politicos, administrativos, económicos $\gamma^{\prime}$ sociales, que han mostrado ser inadccuados. El tercer problenta es el de la rigidez de las instituciones, quc actualmente son incapaces de armonizar los conflictos de interescs a todo nive? para cerrar las brechas entre la oferta y la demanda de recursos energéticos, de matcrias primas y de alimentos".

9. En una visión global de los problemas mundiales actiales, resulta evidente ia utilidad e inclusive la necesiclad de configurar adecundamente las relaciones entre Europa y América Latina. En ambos Consinentes ha habido en las últimas décadas, más que ell otros, un desarrollo de los procesos de integración cconó- 
mica que pueden mutumente beneficiarse de sus respectivas experiencias. Un diálogo curo-latinoamericano más intenso y completo pucde constituir un factor escncial para la major integración c interdependencia global, la misma que se convertirí cada vez más en una nccesidad $y$ en una condición de un desarrollo armónico. Las relaciones entre América Latina y Europa deben constiutir una expresión ejemplar del diálogo cada v'cz más activo entre ei Tercer Mfundo y los paises inclustrializados.

En consecuencia, los participantes, teniendo en cucnta los puntos de vista cxpre. sados por el "Club de Roma" y el "Foro Latino Amcricano", concordaron en:

a) reconocer la utilidad del encucntio por el gran interés de los temas exa. minados $y$ formular volos para que este se repita peribdicamente a fin de continuar, ampliar y profundizar los temas tratados en csta ocasión;

b) expresar su aprecio al ILLA por haber tomacio esta iniciativa y formular votos para que el Instituto Italo-Latino Americano tome, en el ámbito de sus finalidades estatutarias, ulteriores iniciativas a todos los niveles para mantener rivo un diálogo concreto cntre los dos Continentes, que cstán vinculados por tradiciones comunes y quc, en esta fase de los problemas mundiales, con una mayor responsabilidad, tienen gran potencial de complementación, en cspecial, en los campos tccnológico y' cconómico;

c) resaltar los esfuerzos que realiza el Club de Roma - a través del proyecto RIO- para cefinit los medios de aplicación del nucro orclen intcrnacional, que decidió promorer la Asamblea de las Naciones Unidas an los perlodos extraordinarios de sesiones arrila mencionados;

d) Eormular rolos para que profundicen los contactos entrc los sistemas de integración cconómica de Europa y' de América Latina, $y$, en particular, entrc la CEE y al Sistema kconómico Latino Americano (SELA) para hacer posibic, entre otras cosas, un mayor intercambio recíproco de cxperiencias y de coopcración tecnica;

e) reitcrar la aspiración repetidamente manifestada por autorizados voceros latinoamericanos para quc sc realice un encuentro, a nivel gubernativo, entre los paises miembros de la GEE $y$ los paises latinoamericanos;

f) destacar la importancia de la Conferencia sobre la Cooperación Económica 
Internacional en la que un grupo de paises industrializados $y$ de paises en desarrollo están realizando negociaciones en relición con los problemas de la energít, las materias primas, la cooperación internacional y el financiamiento del desarrollo;

g) subrayar la trascendencia de la puesta en marcha de! Sistema Económico Latino-Americano (SELA) como un mecanismo dinínico $y$ flexible para promover in coopcración regional y las relaciones de América Latina con el resto del inundo;

h) tomar nota $y$ agradecer la invitación formulada por el Delegado del Uruguay ante el IrLA para que una segunda edición, organizala por cl IILA, del cncuentro entre el Club de Roma y el Foro Latino Americano se celebre en Montevideo.

\section{PARTICIPANTES}

\section{Club de Roma}

Aureso Peccr. (Italia), Presidente clel "Club de Roma", Presidente de "Italconsult", Roma;

JAN YAN ETTINGER (Holinda), Director del "Bouwcentrum International Edi: cation", Rotterlan;

Jacques Freriond (Suiza), Director del Instituto Universitario de Altos Estudios Internacionales, Ginclira;

Mauricio Gucritre (Francia), Economista y estudioso del desarrollo, Paris;

Edund Prstel (Alemania Federa), "Technische Universitat Hanover", Hanover; Hugo Thlenand (Suiza), Economista y' cnsayista;

RoBerto VACCa (Italia), Profesor y escrilor, Roma.

Hernin Santa Cruz (Chile), Presidente del Centro Internacional de Desarrollo, Paris.

Foro Latino - AMericano

Gabriel V'Aunis (Chilc), cx Ministro de Relaciones Exteriores y Presidente del Foro Latino-Americano;

linRIQUe IcLeshas (Urugua'), Sccretario Ejecutivo de la CEPAL;

Hezro JAGudribt: (Brasil), Profesor y' escritor;

Ronrico Llorisite (Colombia), ex Ministro de Flacienda de Colombia y Puesidente de la "Confederación Latino-Americana de Empresarios";

Cindroo MLendis (Brasil), Presidente de la Universidad "Cíndiclo Mendes" y miembro de la Comisión Central de "Justicia y Paz":

Jame Moxcuro (Ecundor), ex Ministro de Facienda y' Secretario Ejecutivo del SELA;

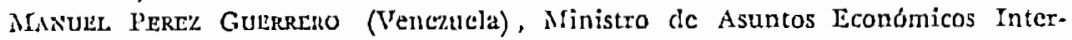
nacionales; 
Lucmo Tomassis (Chile), Director Adjunto del lNYTL y Secretario Ejecutiro de! Foro Latinoamericano.

Mrguel Trionczek (Mréxico). Profesor y Asesor del Consejo Nacional de Ciencias y Tecnología.

\section{ORSIERVADORES}

P^oro Bisocso, Consiglio Nazionale delle Riccrche, Roma;

Bruxo Broledsit, Banca Nazionale del Lavoro, Roma;

Gonzilo Buld Hovos, Organización de las Naciones Unidas para la Agricultura Y' la Alimentación (FAO):

Nestor BekNardo Cucciaruot, Consiglio Nazionale delle Ricerclic, Roma;

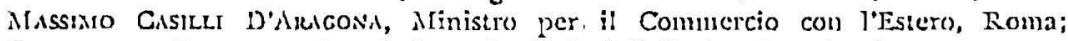
Pinkangelo Cittalano, Associnzione Studi Sociali Latino-Americani;

Giulo Del Bazzo, Centro Italiano di Studi per la Conciliazione Internazionale; Aluhecrer von Cleich, Instituto de Estudios Latino-Americano, Hamburgo;

PiEno Guizarert, CERl'I, Milano;

Hussein Kilulaf Srntok, Liga de los Estaclos Alabes;

V'rtorio MARRAMA, Universití degli Studi, Roma;

El,roxora MIASIN1, World Future Studics Fecieration;

Giuseppe Mrarteuca, Institulo Intemacional pira la Unilicación del Derecho Privaclo (UNIDROIT):

Rrcurdo Monico, Instituto Internacional para la Unilicación del Derecho Prilindo (UNIDROIT);

VINCENzo Rusticheli, Instituto per il Commercio con l'Estero, Roma;

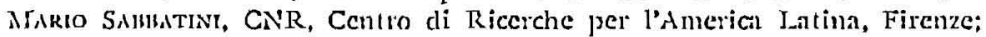

Rinuro SANDr, Instituto per l'Anericn Latint, Africa c Medin Oriente (IIALMO), Romn;

José Mraria SuN Juin, Institulo de Cultura Hispuinica, Madrid;

Frencesco TiNini, Instituto per la Riconstruzione Inklustriale, Roma;

JuAN IGNicio TrN, Instituto de Cistuma His!ńnica, Madrid;

Pasto Trumer, Banco Interimericano de Desamollo;

Nicolò V'ARvest, Ministerio degli Affari Esteri, Roma. 